\title{
Key Prognostic Value of Lysosomal Protein Transmembrane 5 in Kidney Renal Clear Cell Carcinoma
}

\author{
Bin Li, Xue-dong Shi \\ Department of Urology, Yuyao People's Hospital of Zhejiang Province, Ningbo, Zhejiang, 315400, People's Republic of China
}

Correspondence: Xue-dong Shi, Department of Urology, Yuyao People's Hospital of Zhejiang Province, No. 800 Chengdong Road, Yuyao, Ningbo, Zhejiang, 315400, People's Republic of China, Email shixuedongtg@I63.com

Objective: To investigate the significance of lysosomal protein transmembrane 5 (LAPTM5) in kidney renal clear cell carcinoma (KIRC).

Methods: Bioinformatics analysis as an efficient and accurate method was employed to explore the expression levels, prognostic significance, and regulatory pathways of LAPTM5 in KIRC. Finally, the association of LAPTM5 with tumor immune infiltrates was initially investigated.

Results: High LAPTM5 expression was observed in KIRC, and its mRNA expression was correlated with gender, stage, and grade (all $P<0.05$ ) but regardless of age. Besides, high LAPTM5 mRNA expression predicted poor overall survival (OS) of KIRC patients $(P<0.01)$. Further, Cox regression analysis revealed the independent prognostic value of LAPTM5 for OS in KIRC patients $(P<$ 0.001). In addition, the genetic alteration frequency of LAPTM5 was low and had no significant impact on KIRC patient prognosis. However, the low methylation levels of the two methylated sites in the LAPTM5 gene was closely linked to poor OS (all $P<0.05$ ). Kyoto Encyclopedia of Genes and Genomes (KEGG) and gene set enrichment analysis (GSEA) results showed that the common regulatory pathway was immune- and inflammatory-related pathway. Moreover, LAPTM5 was also associated with tumor immune infiltrates (all $P<0.001$ ).

Conclusion: LAPTM5 served as an independent prognostic factor for KIRC patients. LAPTM5 might affect the OS of KIRC patients through the involvement of the immune-related pathway. Therefore, LAPTM5 served as a potential biomarker for OS of KIRC patients.

Keywords: lysosomal protein transmembrane 5, prognosis, regulatory pathway, kidney renal clear cell carcinoma

\section{Introduction}

Renal cell carcinoma (RCC) belongs to one of the most prevalent malignancies worldwide. According to statistics published in 2019, there were approximately 73,820 new cases and 14,770 deaths for RCC in the US. ${ }^{1}$ Kidney renal clear cell carcinoma (KIRC) is the most common subtype of RCC with a proportion of $75-80 \%$, which accounts for the most RCC-related deaths. ${ }^{2}$ It is a type of renal cortical tumor featured with malignant epithelial cells with clear cytoplasm. ${ }^{3}$ Currently, surgery, targeted therapy, and immunotherapy are the preferential methods for the treatment of KIRC due to its resistance to conventional radiotherapy and chemotherapy. ${ }^{3,4}$ However, metastasis still occurred in $30 \%$ of patients who received surgery. ${ }^{5}$ In addition, adaptation to treatment, dynamic variation, and tumor heterogeneity may lead to the limit in the application of the targeted drugs for KIRC treatment. ${ }^{6,7}$ Therefore, there is a strong demand to develop novel biomarkers to improve the long-term survival of this disease.

As a 30-kDa multi-spanning transmembrane protein, lysosomal protein transmembrane 5 (LAPTM5) encodes a membrane protein that localizes to intracellular vesicles, regulating vesicle trafficking. ${ }^{8}$ E3 protein encoded by LAPTM5 might represent an essential role in hematopoiesis. ${ }^{9}$ Additionally, LAPTM5 is preferentially expressed in 
immune cells and hematopoietic cells, and it has a close interaction with Nedd4 family of ubiquitin ligases. LAPTM5 is an active regulator of the proinflammatory signaling pathways in macrophages. ${ }^{10,11}$ Previous studies reported high expression of LAPTM5 in malignant B lymphomas and participated in B cell malignancies, which was involved in negative regulation of $\mathrm{B}$ and $\mathrm{T}$ cell receptor on the cell surface via facilitating lysosome degradation. ${ }^{12,13}$ LAPTM5 deficiency has been shown to activate B cells or T cells due to an increase in level of their respective receptors. ${ }^{14}$ Moreover, knockdown of LAPTM4B, another vital subtype of the LAPTM family inhibited proliferation of prostate, breast and hepatocellular cancers. ${ }^{15-17}$ These results suggested that LAPTM family played an essential role in tumor occurrence and development. Of note, Sui et al have identified that LAPTM5 was one of the key genes in KIRC, ${ }^{18}$ but the underlying mechanism of LAPTM5 involved in KIRC remains clarified.

In this study, public databases and bioinformatics tools were used to evaluate the correlation between LAPTM5 mRNA expression and patient prognosis in KIRC. We proceeded to analyze the genetic alteration and methylation of LAPTM5. Enrichment analyses were conducted to reveal the potential pathways in which LAPTM5 may be involved. Finally, the correlation between LAPTM5 and tumor immune infiltration was studied.

\section{Materials and Methods}

\section{Analysis of the LAPTM5 mRNA Expression}

The gene expression levels of LAPTM5 were assessed using GEPIA, gene set cancer analysis (GSCA), and the University of California Santa Cruz (UCSC) Xena databases. We initially analyzed the LAPTM5 expression in various tumors using the GEPIA database (http://gepia.cancer-pku.cn/) which is a newly developed interactive web server for analyzing the RNA sequencing expression data. As an integrated database for genomic and immunogenomic analyses, GSCA was adopted to explore the LAPTM5 expression in KIRC by inputting "LAPTM5" and selecting "KIRC" in the "expression" column. Moreover, HTseq-FPKM data and phenotype information such as age, gender, and cancer stage for KIRC samples based on GDC TCGA were downloaded from UCSC Xena (https://xenabrowser.net/datapages/) for further analysis. These data were used to further evaluate the LAPTM5 expression in KIRC and paired normal groups. UCSC Xena allows users to explore functional genomic data sets for correlations between genomic and phenotypic variables. Following this, the relation between LAPTM5 mRNA expression and the clinicopathologic characteristics including age, gender, cancer stage, and grade were determined through UALCAN database (http://ualcan.path.uab.edu/analysis.html), which is a comprehensive, user-friendly, and interactive web resource for analyzing OMICS data.

\section{Analysis of the Prognostic Value of LAPTM5 mRNA Expression}

Kaplan-Meier plotter database (http://kmplot.com/analysis/index.php?p=background) was employed to evaluate the prognostic significance of LAPTM5 in KIRC in terms of overall survival (OS) and relapse-free survival (RFS). Then, we examined the effect of LAPTM5 on OS, disease-free interval (DFI), disease-specific survival (DSS), and progressionfree interval (PFI) for KIRC patients based on the GDC TCGA data by the Kaplan-Meier method. Next, the association of LAPTM5 expression with patient clinical outcome with restricted clinicopathological factors was investigated through the Kaplan-Meier plotter website. Subsequently, Cox regression analysis was performed to explore the independent prognostic factors for OS of KIRC patients. Male was set as a reference level for gender, stage 1 for stage, normal for white cell count, hemoglobin, serum calcium, and platelet qualitative.

\section{Genetic Alteration Analysis}

The genetic alteration of LAPTM5 and its relationship with prognosis of KIRC patients were evaluated via cBioPortal database (https://www.cbioportal.org/). This database integrates multidimensional cancer genomics data with interactive analyzing modules for research on gene alteration, co-expression profiles, and survival. The threshold was set as follows: TCGA-KIRC, Firehose Legacy (538 samples), gene, LAPTM5. 


\section{Methylation Analysis}

The UALCAN and DNA methylation interactive visualization database (DNMIVD) (http://119.3.41.228/dnmivd/index/) were applied for analyzing the LAPTM5 promoter methylation profile between KIRC and normal groups. DNMIVD is a comprehensive annotation and interactive visualization database for DNA methylation profile of diverse human cancer constructed with high throughput microarray data. Furthermore, GSCA database was used to explore the correlation between LAPTM5 mRNA expression and its DNA methylation in "Mutation" tab. After that, the prognostic values of LAPTM5 methylation level at different methylated sites were analyzed via MethSurv (https://biit.cs.ut.ee/methsurv/). The survival plots were presented in "Single CPG" module, and the heat map of the relation of LAPTM5 methylation levels with the patient characteristics and gene subregions in "Gene visualization" module. MethSurv is an interactive and user-friendly web portal providing univariate and multivariate survival analysis based on DNA methylation biomarkers using TCGA data.

\section{Identification of Co-Expressed Genes and Functional Enrichment Analysis}

As a database providing a unique platform for biologists and clinicians to access, analyze and compare cancer multi-omics data within and across tumor types, LinkedOmics (http://linkedomics.org/login.php) was adopted to retrieve the genes coexpressed with LAPTM5. The parameters were set as follows: cancer type, TCGA_KIRC; search dataset, RNAseq; dataset attribute, LAPTM5; target dataset, RNAseq; statistic method, Pearson correlation test. According to $\mid \mathrm{z}$ score $\mid, \mathrm{P}<0.001$, false discovery rate (FDR) $<0.01$, top 500 co-expressed genes together with LAPTM5 were loaded into the database for annotation, visualization, and integrated discovery (DAVID) (https://david.ncifcrf.gov/summary.jsp) for gene ontology (GO) and Kyoto Encyclopedia of Genes and Genomes (KEGG) analyses.

\section{Gene Set Enrichment Analysis (GSEA)}

GSEA was performed to reveal the pathological role of LAPTM5 in KIRC. The parameters were set as follows: number of permutations (1000), permutations type (gene_set), enrichment statistic (weighted), metric for ranking genes (Pearson), gene list ordering mode (descending). The terms with $F D R<0.25$ and $P<0.05$ were considered as significant pathways.

\section{Immune Infiltrates Analysis}

Tumor immune estimation resource (TIMER) web server (https://cistrome.shinyapps.io/timer/) is a comprehensive resource for systemic analysis of immune infiltrates across 32 cancer types. The abundance of six immune infiltrates including B cells, CD4 $+\mathrm{T}$ cells, CD8 $+\mathrm{T}$ cells, neutrophils, macrophages, and dendritic cells are estimated using the TIMER algorithm. The correlation between LAPTM5 expression and infiltration of immune cells in KIRC were examined in the "Gene" module of TIMER.

\section{Statistical Analysis}

Statistical analyses were performed by SPSS23.0 (SPSS, Inc., Chicago, IL, USA) software. The differential expression of LAPTM5 between KIRC and paired normal groups was evaluated using paired $t$-test. Kaplan-Meier survival analyses were performed using the Log rank test. Spearman correlation and Pearson correlation coefficient were utilized to measure the linear dependence between variables in GSCA and LinkedOmics, respectively. $P$ value less than 0.05 was considered as statistically significant.

\section{Results}

\section{Expression Analysis of LAPTM5 in KIRC}

The LAPTM5 mRNA expression in various cancers was firstly analyzed and the results are shown in Figure 1A. Then, we examined the mRNA expression of LAPTM5 in KIRC, and observed that the transcriptional level of LAPTM5 was significantly elevated in KIRC in comparison to the normal group $(F D R<0.001)$ (Figure 1B). Moreover, analysis of the 


\section{A}

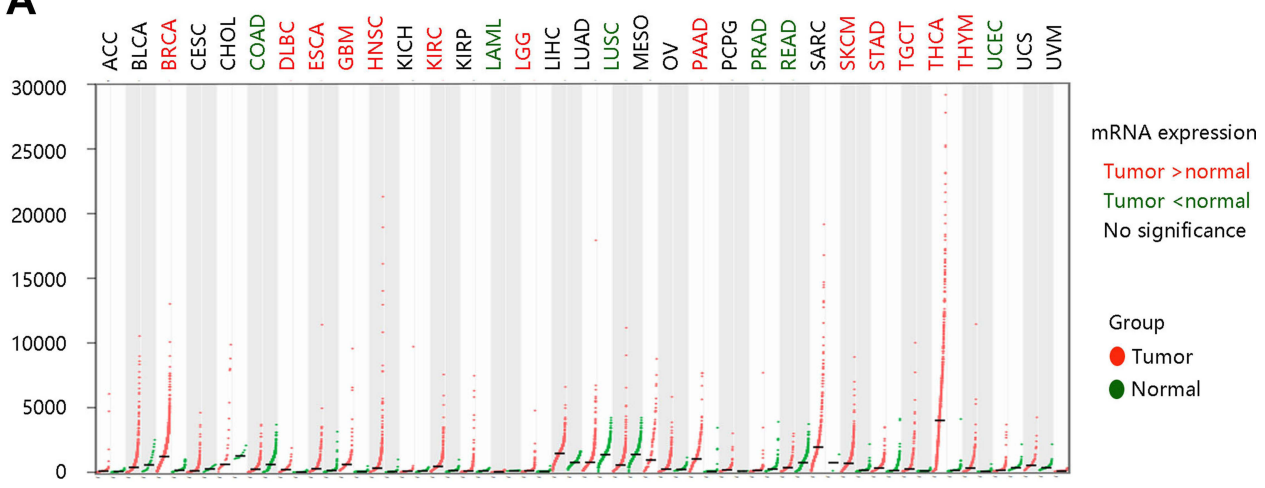

B

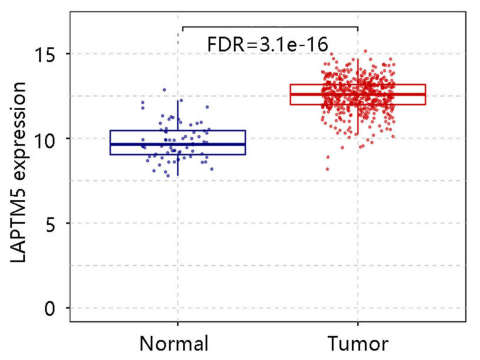

C

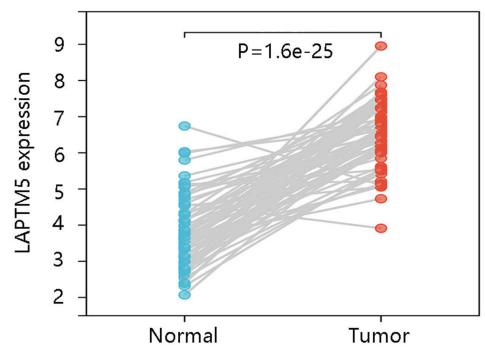

Figure I LAPTM5 overexpression in KIRC. (A) LAPTM5 mRNA expression in multiple cancers. (B) LAPTM5 mRNA expression between KIRC and normal groups. FDR, false discovery rate. (C) LAPTM5 mRNA expression in a paired comparison of KIRC and their adjacent normal tissues.

RNAseq data of GDC TCGA-KIRC samples exhibited overexpression of LAPTM5 in KIRC tissues compared to paired normal controls $(P<0.001)$ (Figure $1 \mathrm{C})$. These results suggested that LAPTM5 was overexpressed in KIRC.

After a significant difference of LAPTM5 mRNA expression between normal and KIRC groups has been observed, the relationship between clinicopathological characteristics and its mRNA expression was next explored. As presented in

A

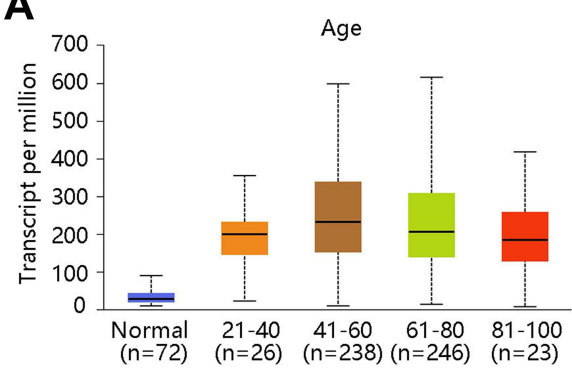

C

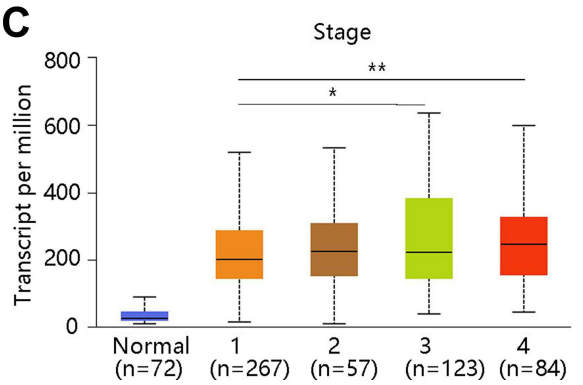

B

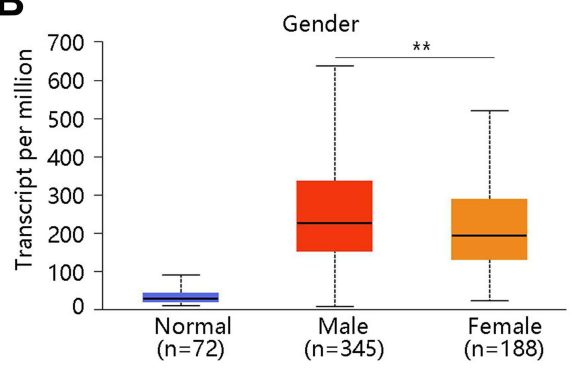

D

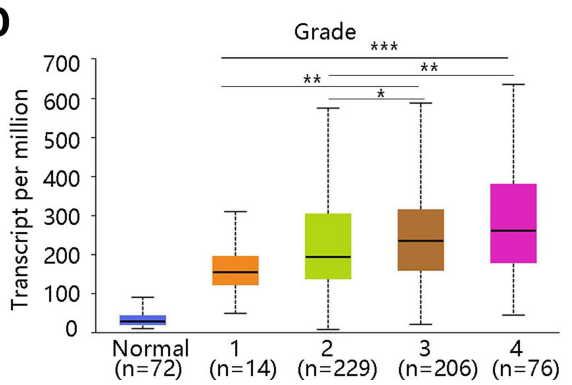

Figure 2 The relation between LAPTM5 mRNA expression and clinicopathological characteristics in UALCAN. (A) Age. (B) Gender. (C) Stage. (D) Grade. *P < 0.05, **P < 0.0 I, **** $\mathrm{P}<0.001$. 
Figure 2A, no significant relation was found between LAPTM5 mRNA expression and age. Nevertheless, LAPTM5 mRNA expression was significantly connected with gender, cancer stage, and grade (all $P<0.05$ ) (Figure 2B-D).

\section{High LAPTM5 mRNA Expression Was Related to Poor OS of KIRC Patients}

To investigate the prognostic significance of LAPTM5 mRNA expression in KIRC, we determined the correlation between LAPTM5 and patient OS, and RFS. The KIRC patients who possessed a low LAPTM5 mRNA expression tended to have better prognosis $(P<0.01)$ (Figure 3A), but there was no significant impact of LAPTM5 on RFS (Figure 3B). Based on GDC TCGA-KIRC data, high LAPTM5 mRNA expression resulted in worse OS $(P<0.01)$ (Figure 3C). However, high LAPTM5 mRNA expression was not significantly linked to the DFI, DSS, and PFI (Figure 3D-F). These findings revealed that LAPTM5 was a potential biomarker for OS in KIRC patients.

To further determine the prognostic value of LAPTM5 mRNA expression in KIRC, Kaplan-Meier plotter website was employed to evaluate the correlation between LAPTM5 mRNA expression and patient prognosis stratified by the clinical parameters. As presented in Table 1, LAPTM5 was negatively related to OS among stage 1 , and stage 4 patients $(P<0.05)$. Similarly, a negative correlation was found between LAPTM5 and OS in both female and male patients $(P<0.05)$. Besides, high LAPTM5 mRNA expression led to poor OS in Caucasian patients $(P<0.05)$. However, LAPTM5 was not notably associated with RFS in KIRC patients with restricted clinicopathological characteristics. The above results indicated that

A
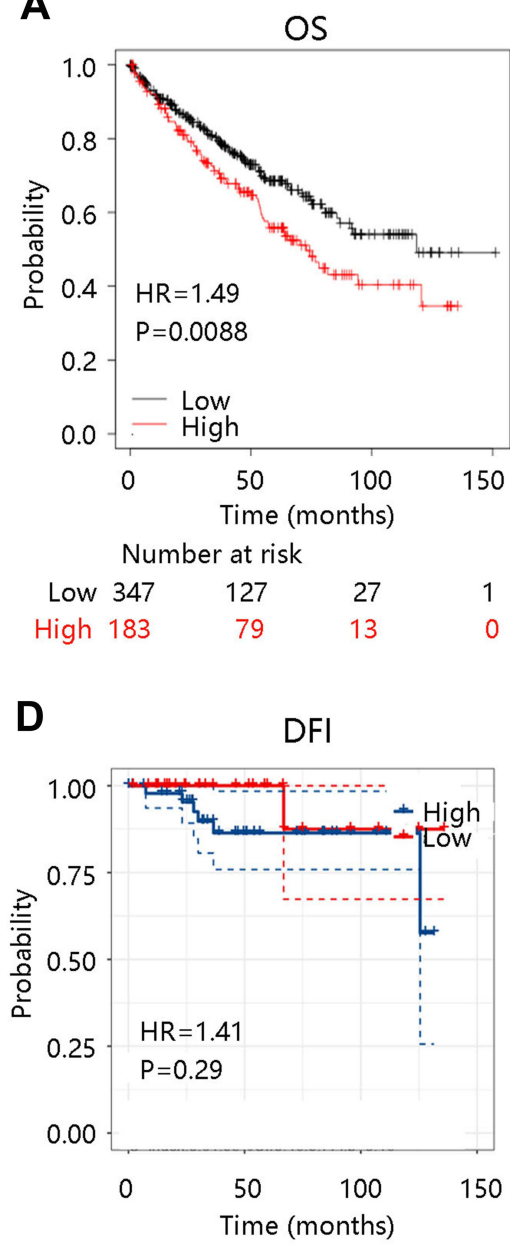

B

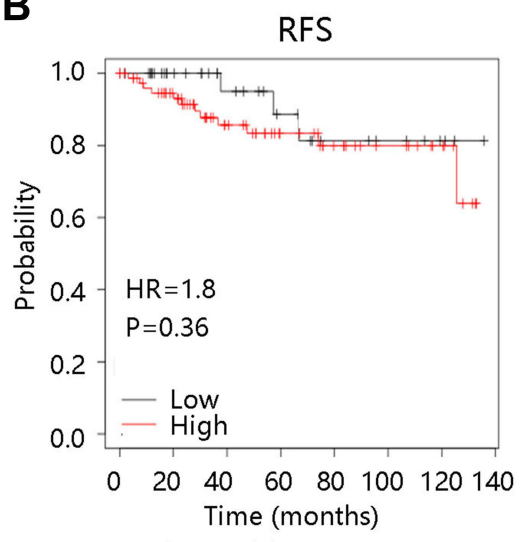

Number at risk

Low $\begin{array}{llllllll}37 & 27 & 19 & 13 & 8 & 6 & 3 & 0\end{array}$

High $80 \begin{array}{lllllll}61 & 40 & 27 & 19 & 13 & 8 & 0\end{array}$

E
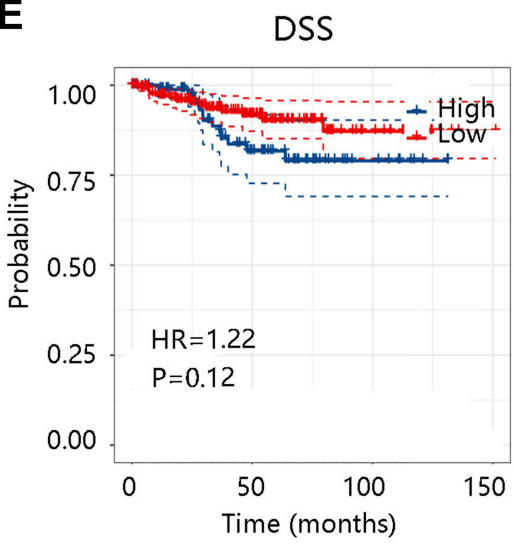

$\begin{array}{ccccc}\text { High } & 91 & 39 & 9 & 0 \\ & 191 & 73 & 14 & 1\end{array}$
C

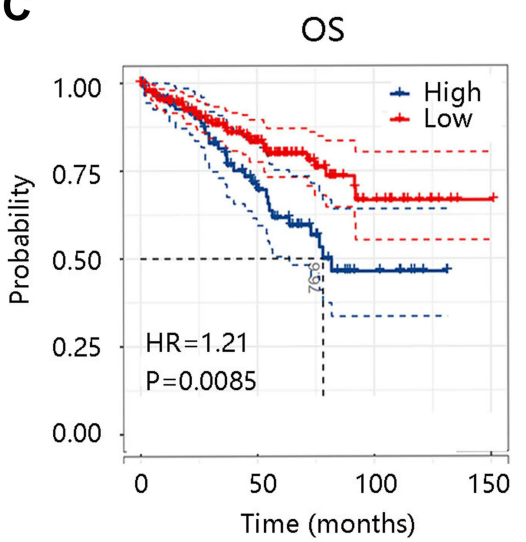

$\begin{array}{lllll}\text { High } & 86 & 37 & 8 & 0\end{array}$

Low $200 \quad 77 \quad 15 \quad 1$

$\mathbf{F}$

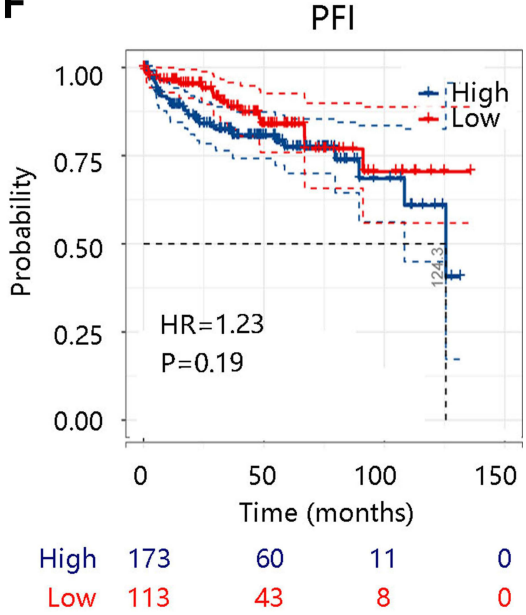

Figure 3 Correlation between LAPTM5 and KIRC patient prognosis. (A) OS and (B) RFS in Kaplan-Meier plotter. (C) OS, (D) DFI, (E) DSS, and (F) PFI based on GDC TCGA data.

Abbreviations: OS, overall survival; RFS, relapse-free survival; DFI, disease-free interval; DSS, disease-specific survival; PFI, progression-free interval; HR, hazard ratio. 
Table I The Correlation Between LAPTM5 mRNA Expression and Prognosis in KIRC Patients with Restricted Pathological Factors

\begin{tabular}{|c|c|c|c|c|c|c|}
\hline \multirow[t]{2}{*}{ Clinicopathological Characteristics } & \multicolumn{3}{|c|}{ Overall Survival } & \multicolumn{3}{|c|}{ Relapse-Free Survival } \\
\hline & Number & HR (95\% Cl) & P-value & Number & HR (95\% Cl) & P-value \\
\hline \multicolumn{7}{|l|}{ Stage } \\
\hline I & 265 & $2.26(1.25-4.09)$ & 0.0055 & 73 & $0.38(0.04-3.7)$ & 0.39 \\
\hline 2 & 57 & $0.7(0.19-2.55)$ & 0.59 & 18 & l & l \\
\hline 3 & 123 & $0.52(0.24-1.1)$ & 0.081 & 25 & $0.32(0.03-3.87)$ & 0.35 \\
\hline 4 & 82 & $2(1.14-3.52)$ & 0.014 & 0 & l & l \\
\hline \multicolumn{7}{|l|}{ Grade } \\
\hline 1 & 14 & l & l & 4 & l & l \\
\hline 2 & 227 & $1.62(0.88-2.98)$ & 0.12 & 61 & $2.45(0.27-21.97)$ & 0.41 \\
\hline 3 & 206 & $0.63(0.37-1.06)$ & 0.078 & 41 & I.8I (0.35-9.3I) & 0.47 \\
\hline 4 & 75 & $1.36(0.77-2.39)$ & 0.29 & 7 & I & I \\
\hline \multicolumn{7}{|l|}{ Gender } \\
\hline Female & 186 & $1.7(I .02-2.8 I)$ & 0.038 & 49 & $0.46(0.05-4.4)$ & 0.49 \\
\hline Male & 344 & $1.55(1.03-2.35)$ & 0.034 & 67 & $0.58(0.18-1.93)$ & 0.37 \\
\hline \multicolumn{7}{|l|}{ Race } \\
\hline Caucasian & 459 & $1.5(1.09-2.04)$ & 0.011 & 83 & $2.26(0.5-10.23)$ & 0.28 \\
\hline Asian & 8 & I & I & 0 & I & I \\
\hline African-American & 66 & $1.84(0.55-6.12)$ & 0.31 & 34 & 0 (0-Inf) & 0.24 \\
\hline
\end{tabular}

Note: / Values indicated the sample number too low for meaningful analysis.

Abbreviations: KIRC, kidney renal clear cell carcinoma; HR, hazard ratio; $95 \% \mathrm{Cl}, 95 \%$ confidence interval.

high expression level of LAPTM5 affected the OS of KIRC patients particularly in stage 1, stage 4, and Caucasian patients with regardless of gender.

To explore the independent prognostic predictors for OS of KIRC patients, LAPTM5 and clinicopathological factors were integrated into Cox regression analyses. In univariate analysis, age, stage 3, stage 4, low hemoglobin, elevated serum calcium and platelet qualitative, and LAPTM5 were significantly related to prognosis of KIRC patients $(P<0.01)$ (Table 2). In multivariate analysis, only age, stage 4, and LAPTM5 had statistical differences (all $P<0.05$ ) (Table 3), suggesting that LAPTM5 served as an independent prognostic predictor for OS of KIRC patients.

\section{Genetic Alteration Frequency of LAPTM5 Was Low and Not Associated with the Prognosis in KIRC}

To investigate the value of LAPTM5 genetic alteration in KIRC, the type and proportion of LAPTM5 genetic alteration were evaluated through cBioPortal. We observed that the genetic alteration was detected in $4 \%$ of the patients, and the alteration type was mRNA high (Figure 4A). After that, we analyzed the correlation between LAPTM5 mRNA expression and prognosis. There was no significant difference in OS and disease-free survival (DFS) between altered and unaltered groups (Figure 4B and C). The above findings implied that genetic alteration frequency of LAPTM5 was low and not linked to patient prognosis.

\section{Hypomethylation of LAPTM5 Was Linked to Worse OS of KIRC Patients}

To probe into the role of LAPTM5 methylation in KIRC, we first analyzed the LAPTM5 methylation status and observed hypomethylation of LAPTM5 in the promoter region in KIRC by UALCAN and DNMIVD databases (all $P<0.001$ ) (Figure 5A and B). Subsequently, the correlation between LAPTM5 methylation and its mRNA expression was evaluated through GSCA. As shown in Figure 5C, LAPTM5 methylation exhibited a strong negative relation with its mRNA expression in KIRC with a Cor. $=-0.59(F D R<0.001)$.

Following this, we extracted the prognosis-related methylated sites of the LAPTM5 gene using the MethSurv database. The genomic regions were classified into TSS 1500, 3'UTR, first exon, 5'UTR, body, and TSS 200. ${ }^{19}$ Figure 5D demonstrates the heat map of the relation of LAPTM5 methylation levels with the patient characteristics 
Table 2 Univariate Analysis of the Correlation of LAPTM5 and Other Clinical Risk Factors with OS in KIRC Patients

\begin{tabular}{|l|l|l|l|}
\hline Covariates & HR & $\mathbf{9 5 \%} \mathbf{~ C I}$ & P-value \\
\hline Age & 1.03 I & $1.018-1.044$ & $<0.00$ I \\
Female & 1.007 & $0.729-1.392$ & 0.964 \\
Stage 2 & 1.415 & $0.752-2.664$ & 0.282 \\
Stage 3 & 2.868 & $1.859-4.423$ & $<0.00$ I \\
Stage 4 & 6.747 & $4.496-10.126$ & $<0.00$ I \\
White cells count (low) & 1.673 & $0.680-4.115$ & 0.262 \\
White cells count (elevated) & 0.721 & $0.500-1.041$ & 0.08 I \\
Hemoglobin (low) & 2.117 & $1.465-3.060$ & $<0.00$ I \\
Hemoglobin (elevated) & 3.668 & $0.880-15.288$ & 0.074 \\
Serum calcium (low) & 0.779 & $0.543-1.116$ & 0.173 \\
Serum calcium (elevated) & 3.325 & $1.573-7.029$ & 0.002 \\
Platelet qualitative (low) & 1.433 & $0.855-2.403$ & 0.172 \\
Platelet qualitative (elevated) & 3.517 & $2.319-5.336$ & $<0.00$ I \\
LAPTM5 & 5.826 & $3.331-10.192$ & $<0.00$ I \\
\hline
\end{tabular}

Abbreviations: OS, overall survival; KIRC, kidney renal clear cell carcinoma; $\mathrm{HR}$, hazard ratio; $95 \% \mathrm{Cl}, 95 \%$ confidence interval.

Table 3 Multivariate Analysis of the Correlation of LAPTM5 and Other Clinical Risk Factors with OS in KIRC Patients

\begin{tabular}{|l|l|l|l|}
\hline Covariates & HR & $\mathbf{9 5 \%} \mathbf{C l}$ & P-value \\
\hline Age & 1.065 & $1.030-1.100$ & $<0.00$ I \\
Female & 1.318 & $0.657-2.646$ & 0.437 \\
Stage 2 & 0.982 & $0.25 I-3.846$ & 0.979 \\
Stage 3 & 1.558 & $0.683-3.555$ & 0.292 \\
Stage 4 & 5.215 & $1.435-18.948$ & 0.012 \\
White cells count (low) & 2.742 & $0.321-23.408$ & 0.356 \\
White cells count (elevated) & 1.467 & $0.566-3.802$ & $0.43 \mathrm{I}$ \\
Hemoglobin (low) & 1.437 & $0.599-3.447$ & 0.416 \\
Hemoglobin (elevated) & 3.178 & $0.300-33.619$ & 0.337 \\
Serum calcium (low) & 0.995 & $0.406-2.437$ & $0.99 \mathrm{I}$ \\
Serum calcium (elevated) & 3.375 & $0.404-28.184$ & $0.26 \mathrm{I}$ \\
Platelet qualitative (low) & 1.430 & $0.516-3.963$ & 0.492 \\
Platelet qualitative (elevated) & 1.729 & $0.613-4.880$ & $0.30 \mathrm{I}$ \\
LAPTM5 & 4.765 & $2.453-9.256$ & $<0.00 \mathrm{I}$ \\
\hline
\end{tabular}

Abbreviations: OS, overall survival; KIRC, kidney renal clear cell carcinoma; HR, hazard ratio; $95 \% \mathrm{Cl}$, $95 \%$ confidence interval.

and gene subregions. By assessing all 10 methylation sites of LAPTM5 in KIRC, we found that two hypomethylation sites LAPTM5-3'UTR-Open_Sea-cg13246468 and LAPTM5-Body-Open_Sea-cg19919590 predicted worse clinical outcome of KIRC patients $(\mathrm{P}<0.05)$ (Figure 5E and F), suggesting that hypomethylation of LAPTM5 resulted in poor OS of KIRC patients.

\section{Identification and Enrichment Analysis of LAPTM5 and Its Co-Expressed Genes}

To reveal the underlying mechanism of LAPTM5 in KIRC, LinkedOmics was firstly utilized to retrieve the genes coexpressed with LAPTM5 in KIRC. The volcano plot presented the association results using Pearson Correlation Coefficient (Figure 6A). The top 50 positively and negatively correlated genes were exhibited in the heat maps (Figure 6B and C). Then, the top 500 co-expressed genes together with LAPTM5 were loaded into DAVID database for GO and KEGG analyses. For biological processes, LAPTM5 and its co-expressed genes were mainly enriched in 


\section{A}

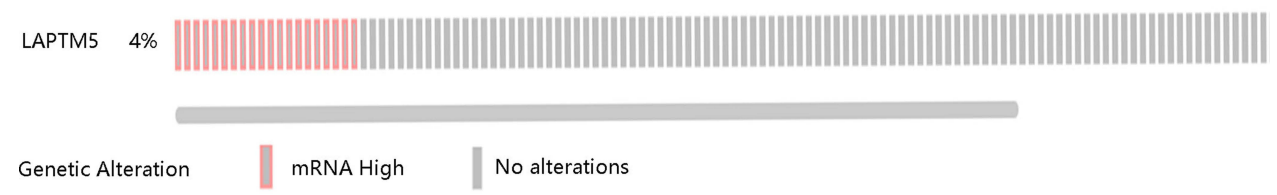

B
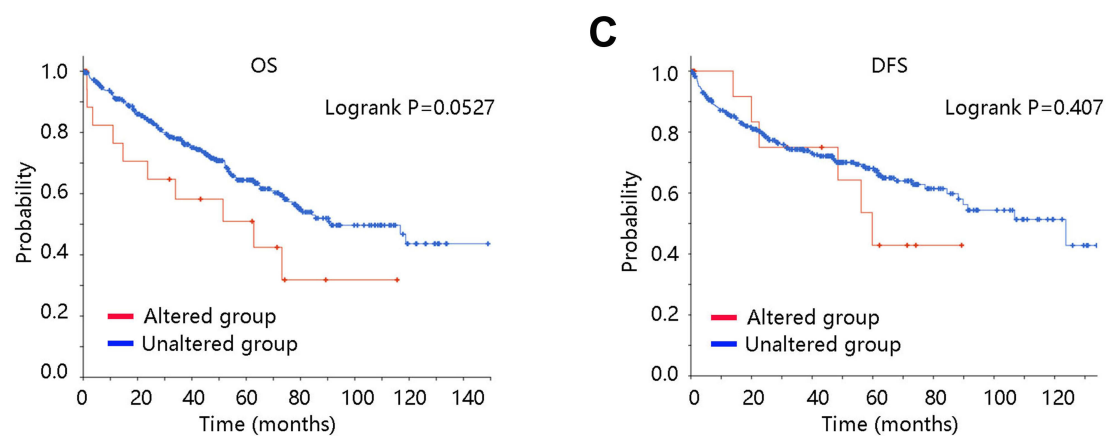

Figure 4 The genetic alteration frequency of LAPTM5 was low and not associated with prognosis in KIRC patients. (A) The genetic alteration frequency of LAPTM5. The correlation between LAPTM5 gene alteration and (B) OS, and (C) DFS.

Abbreviations: OS, overall survival; DFS, disease-free survival.

signal transduction, immune response, and inflammatory response (Figure 6D). For cellular components, they were mainly involved in plasma membrane, integral component of membrane, and integral component of plasma membrane (Figure 6E). For molecular functions, they were mainly participated in receptor activity, transmembrane signaling receptor activity, and carbohydrate binding (Figure 6F). The major KEGGs were cell adhesion molecules, cytokinecytokine receptor interaction, and chemokine signaling pathway (Figure 6G). Notably, the pathways in which LAPTM5 and its co-expressed genes were mainly involved were immune- and inflammatory-related pathways. These results indicated that LAPTM5 might be related to cancer progression.

\section{GSEA}

To further elucidate the pathological role of LAPTM5 in KIRC, GSEA was performed to identify the LAPTM5 associated signaling pathways in KIRC. The results showed that gene sets differentially enriched in the high LAPTM5 expression phenotype associated with immunomodulation, such as antigen processing and presentation, cytokinecytokine receptor interaction, natural killer cell mediated cytotoxicity, Toll like receptor signaling pathway, JAK-STAT signaling pathway, and cell adhesion molecules (Figure 7). The detailed information of these six most significant pathways are presented in Table 4. Combined with the functional enrichment results, we found that these top six significant pathways were the consistent pathways. Thus, LAPTM5 might be involved in the development and progression of KIRC via regulating immune- and inflammatory related pathways.

\section{Association of LAPTM5 Expression with Immune Infiltrates}

Due to the involvement of LAPTM5 in the immune-related signaling pathways, we next focused on the relation between LAPTM5 expression and immune infiltration level in KIRC through TIMER database. As shown in Figure 8, LAPTM5 was positively associated with all the immune infiltrates including B cell, CD8+ T cell, CD4+ T cell, macrophage, neutrophil, and dendritic cell (all $P<0.001$ ), indicating that LAPTM5 was closely related to immune cell infiltration.

\section{Discussion}

The discovery of important tumor biomarkers by bioinformatics analysis has become a reliable method, which provides various information such as expression, prognosis, and regulatory pathways. ${ }^{20,21}$ In this study, we evaluated the expression and prognostic significance of LAPTM5 in KIRC based on bioinformatics approaches. Various public databases were employed to eliminate any discrepancies in the LAPTM5 expression and its prognostic value in KIRC. 

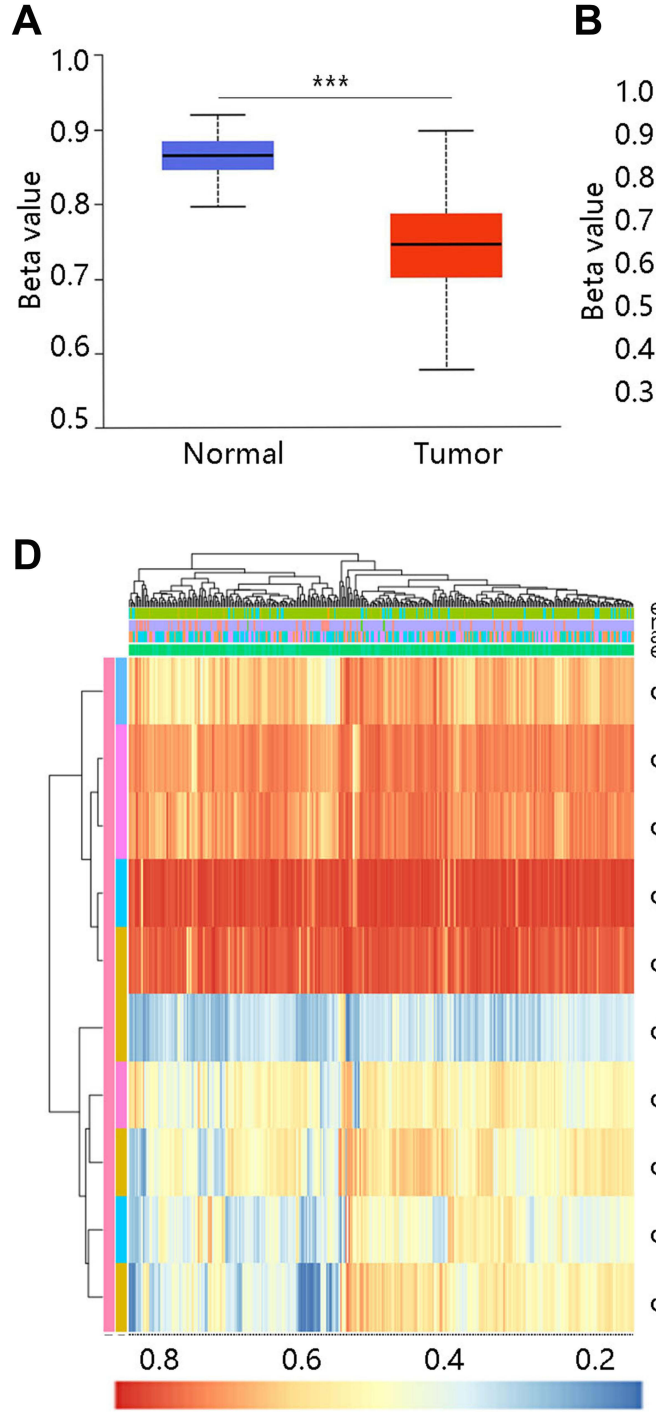

B

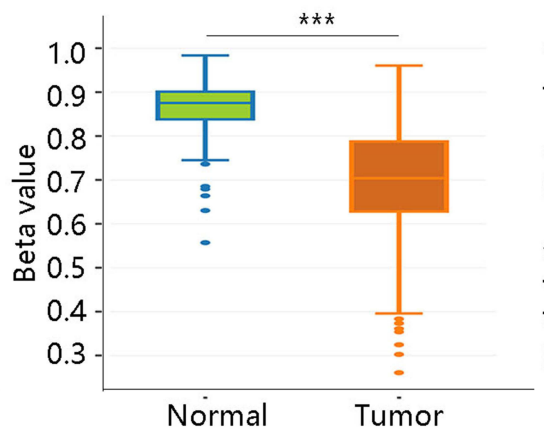

C

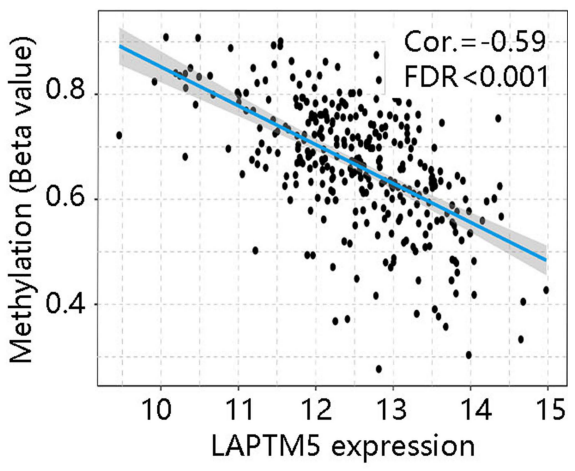

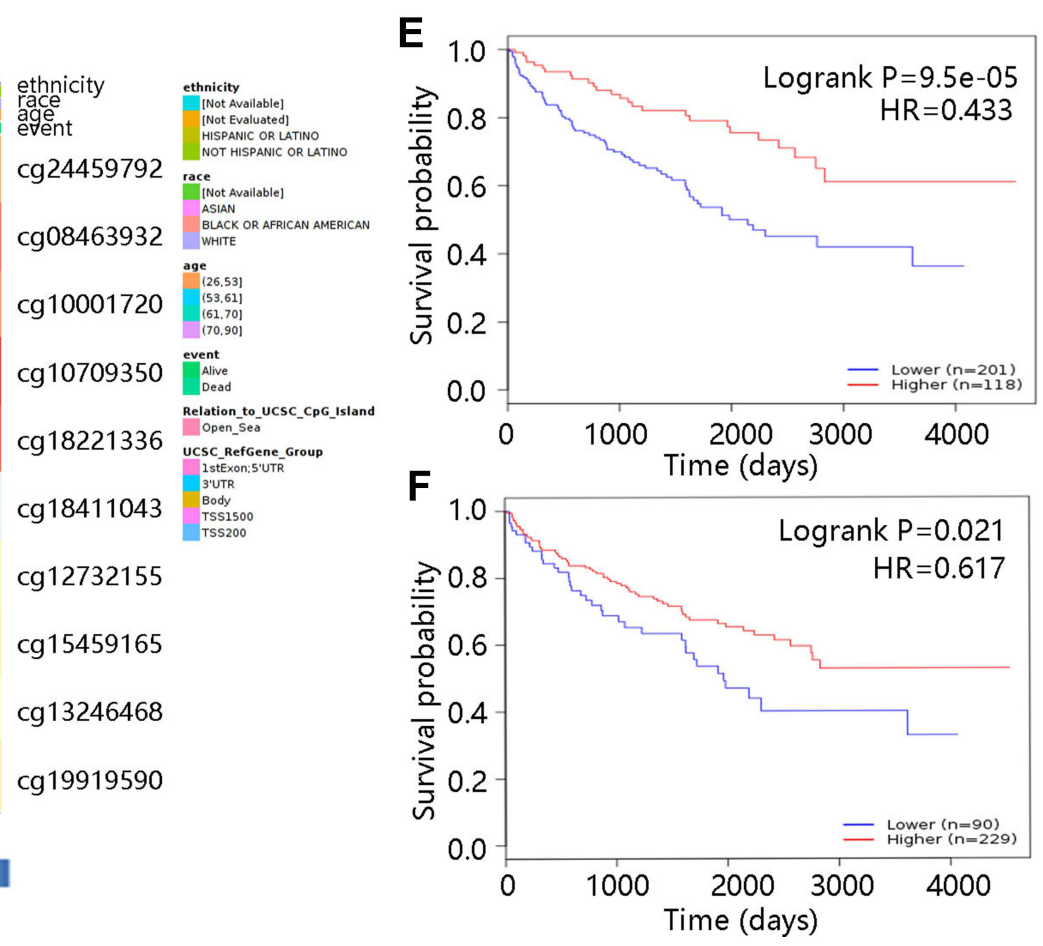

Figure 5 Methylation of LAPTM5 was related to prognosis of KIRC patients. Methylation status of LAPTM5 in (A) UALCAN and (B) DNMIVD. (C) The correlation between LAPTM5 methylation and its mRNA expression. (D) The heat map of the relation of LAPTM5 methylation levels with the patient characteristics and gene subregions. Association of methylation at (E) 3'UTR-Open_Sea-cgl3246468 and (F) Body-Open_Sea-cg199/9590 with KIRC patient OS.

Abbreviations: FDR, false discovery rate; HR, hazard ratio.

The results showed a consistent association of high LAPTM5 mRNA expression with unfavorable clinical outcome of KIRC patients. The genetic alteration and methylation of LAPTM5 were also explored to further understand the role of LAPTM5 in KIRC. Finally, the enrichment analyses revealed that LAPTM5 was mainly involved in immune- and inflammation-related pathways.

There is increasing evidence showed that LAPTM5 was related to multiple tumors. Low mRNA expression of LAPTM5 predicted worse prognosis of patients with non-small cell lung cancer and esophageal squamous cell carcinoma. ${ }^{22}$ Downregulation of LAPTM5 inhibited the proliferation and viability of bladder cancer cells. ${ }^{23}$ Besides, LAPTM5 mRNA expression was elevated in testicular germ cell tumors, and its high expression contributed to poor OS. ${ }^{9}$ LAPTM5 also served as a biomarker for patients with lung cancer, exhibiting the correlation between LAPTM5 and methylation. ${ }^{24}$ Additionally, LAPTM5 mRNA expression was decreased in neuroblastoma cell lines and primary tumors by DNA methylation around its transcriptional start site. ${ }^{25}$ Taken together, LAPTM5 represented a crucial role in the cancer progression. In this study, we observed that LAPTM5 was highly expressed in KIRC, and its expression was 


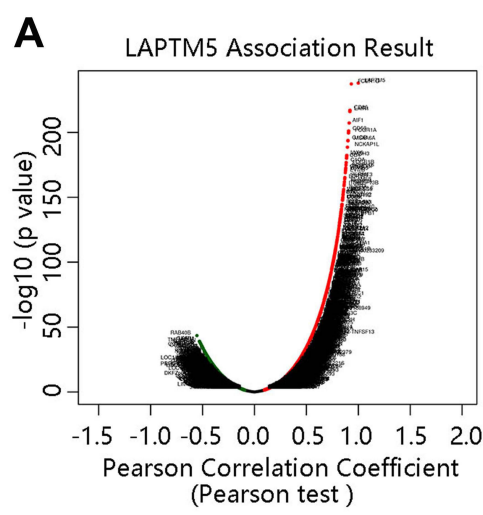

D

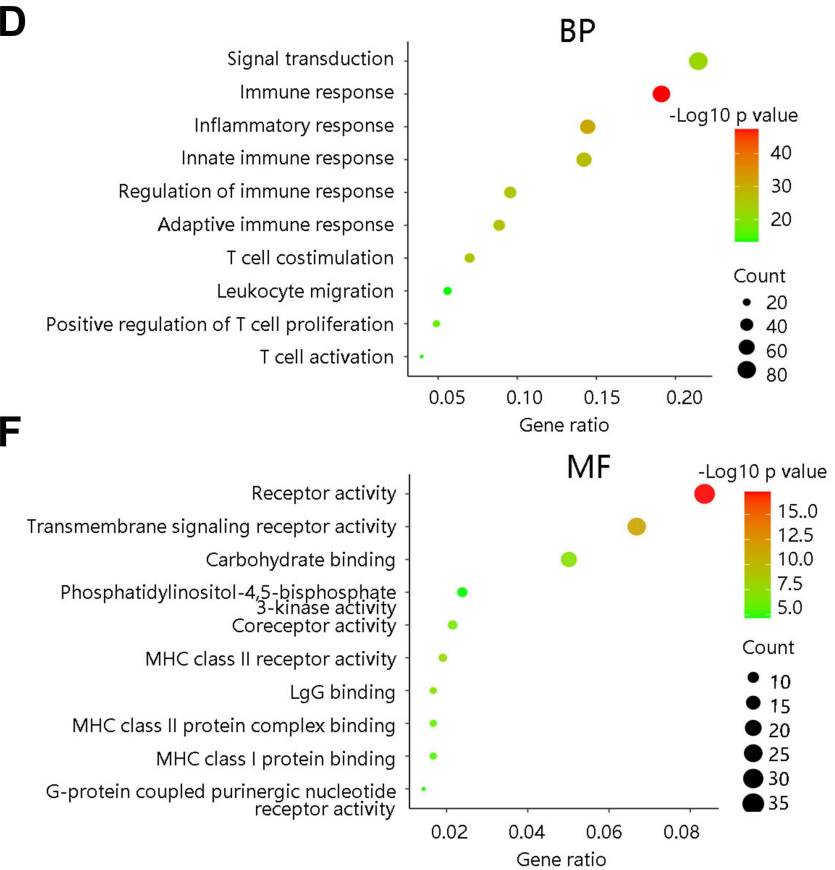

B Top 50 positively correlated genes

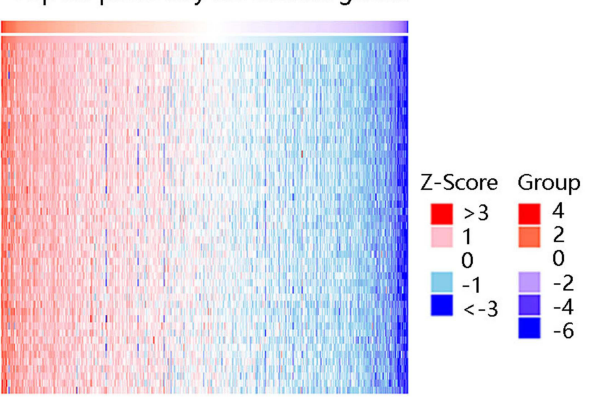

C Top 50 negatively correlated genes

E

E

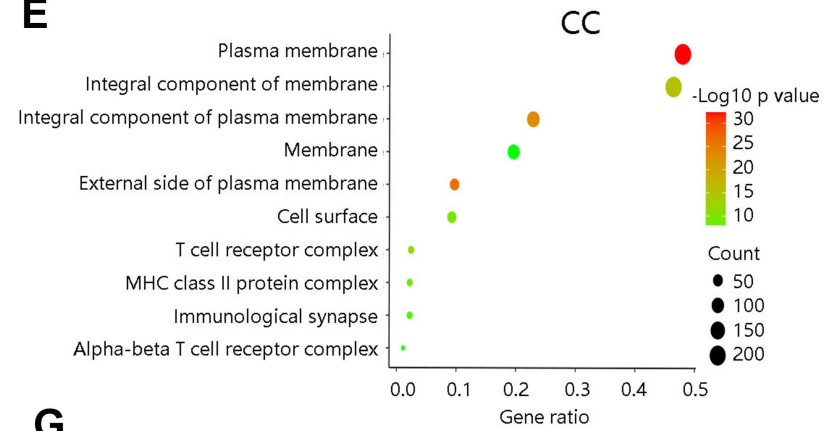

G

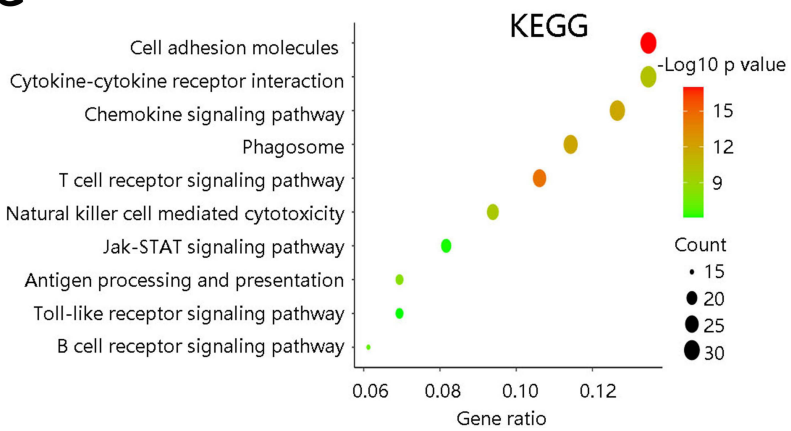

Figure 6 Identification and functional enrichment analyses of genes co-expressed with LAPTM5. (A) The volcano plot of genes co-expressed with LAPTM5. (B) The top 50 positively correlated genes. (C) The top 50 negatively correlated genes. (D) BP, (E) CC, (F) MF, and (G) KEGG pathway enrichment of LAPTM5 and its co-expressed genes. Abbreviations: BP, biological process; CC, cellular component; MF, molecular function; KEGG, Kyoto Encyclopedia of Genes and Genomes.

significantly related to gender, stage, and grade. Based on Kaplan-Meier plotter analysis, we found that high transcriptional level of LAPTM5 contributed to worse OS of KIRC patients, particularly by affecting OS of patients at stage 1, stage 4, and Caucasian. Further Cox regression analysis revealed the independent prognostic value of LAPTM5 for OS in KIRC, indicating that LAPTM5 might be a potential prognostic biomarker for this tumor.

We next embarked our research on the genetic alteration of LAPTM5 and its impact on patient prognosis. Despite the genetic alterations in LAPTM5 were observed, they were less frequent and not significantly associated with OS or DFS of the KIRC patients. Following this, the methylation of LAPTM5 and its relation to the clinical outcome were investigated. Previous studies have pointed out the essential role of LAPTM5 methylation status in tumors. Cortese et al have reported that the hypermethylation of LAPTM5 in poorly differentiated lung cancers in comparison to moderately differentiated lung cancers. ${ }^{24}$ Besides, the silencing of LAPTM5 by hypermethylation might result in the development of more aggressive cancer phenotypes in myeloma cells. ${ }^{26}$ While in our study, hypomethylation of LAPTM5 at two sites led to poor OS of KIRC patients. Thus, it is reasonable to speculate that LAPTM5 overexpression in KIRC and contributed to shorter survival time of the patients due to its hypomethylation.

To determine the function of LAPTM5, the genes co-expressed with LAPTM5 were screened for functional enrichment analysis. Major KEGG pathways modulated by LAPTM5 co-expressed genes were cell adhesion molecules, 
Antigen processing and presentation

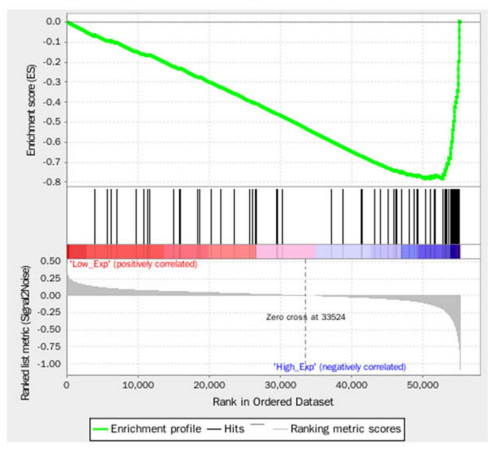

Toll like receptor signaling pathway

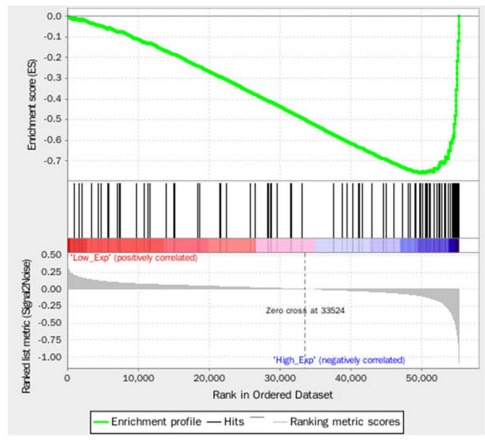

Cytokine-cytokine receptor interaction

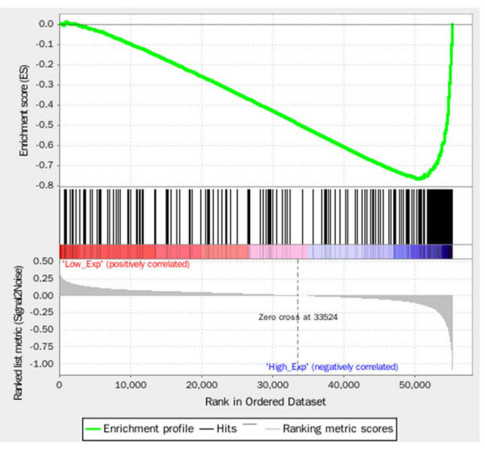

JAK-STAT signaling pathway

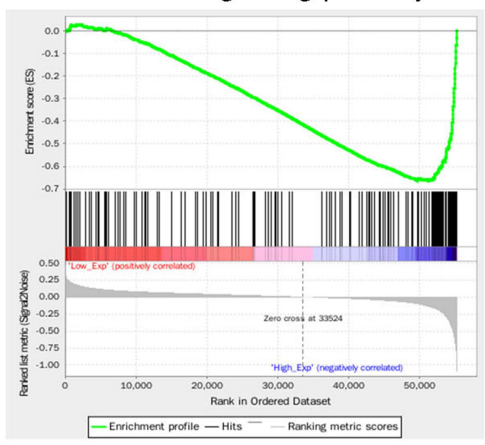

Natural killer cell mediated cytotoxicity

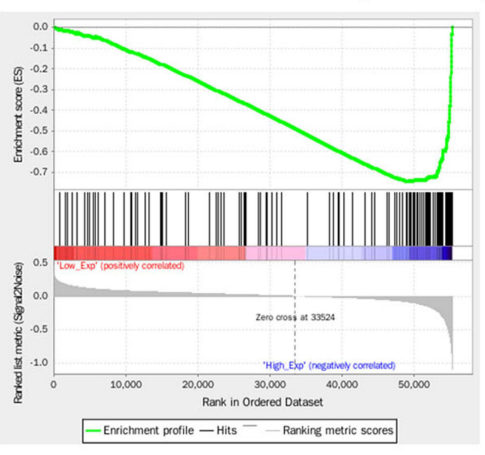

Cell adhesion molecules

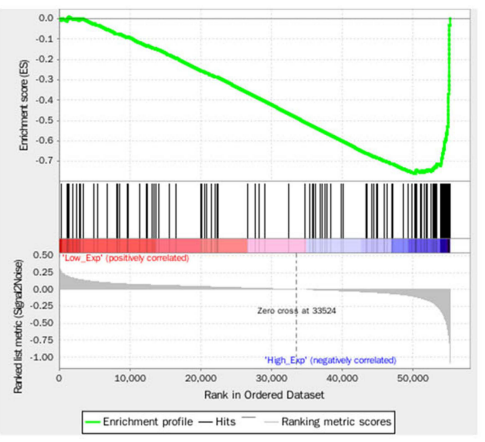

Figure 7 Gene sets enriched in the high LAPTM5 expression phenotype by GSEA.

cytokine-cytokine receptor interaction, and chemokine signaling pathway. Moreover, GSEA results showed six most significantly enriched pathways in high expression phenotype including JAK-STAT signaling pathway, which played a regulatory role in cell proliferation, and differentiation, and migration. ${ }^{27}$ All these pathways were correlated with immune and inflammation, suggesting that LAPTM5 might be involved in the KIRC progression by regulating immunerelated pathway. The pathway results triggered our interest to investigate the correlation between LAPTM5 and immune infiltration. Interestingly, LAPTM5 had robust relation with tumor immune infiltrates, but the detailed underlying mechanism required further exploration. Future research is also needed to explore the LAPTM5-targeted drugs, as LAPTM5 may be a valuable biomarker for KIRC.

In conclusion, LAPTM5 was highly expressed in KIRC, and its high mRNA level might lead to worse prognosis of KIRC patients through regulation of immune- and inflammation-related pathways. Moreover, we found the methylation

Table 4 Gene Sets Enriched in the High LAPTM5 Expression Phenotype

\begin{tabular}{|l|l|l|l|l|}
\hline KEGG Name & ES & NES & Nominal p-value & FDR q-value \\
\hline Antigen processing and presentation & -0.78396684 & -2.7777734 & $<0.00 I$ & $<0.00 \mathrm{I}$ \\
Cytokine-cytokine receptor interaction & -0.79863626 & -2.6519237 & $<0.00 \mathrm{I}$ & $3.29 \mathrm{E}-04$ \\
Natural killer cell mediated cytotoxicity & $-0.74516 \mathrm{I}$ & -2.630032 & $<0.00 \mathrm{I}$ & $1.97 \mathrm{E}-04$ \\
Toll like receptor signaling pathway & -0.7601358 & -2.5928254 & $<0.00 \mathrm{I}$ & $1.4 \mathrm{IE}-04$ \\
JAK-STAT signaling pathway & -0.66569597 & -2.5872974 & $<0.00 \mathrm{I}$ & $1.23 \mathrm{E}-04$ \\
Cell adhesion molecules & -0.7613634 & -2.5573685 & $<0.00 \mathrm{I}$ & $2.07 \mathrm{E}-04$ \\
\hline
\end{tabular}

Abbreviations: ES, enrichment score; NES, normalized enrichment score; FDR, false discovery rate. 

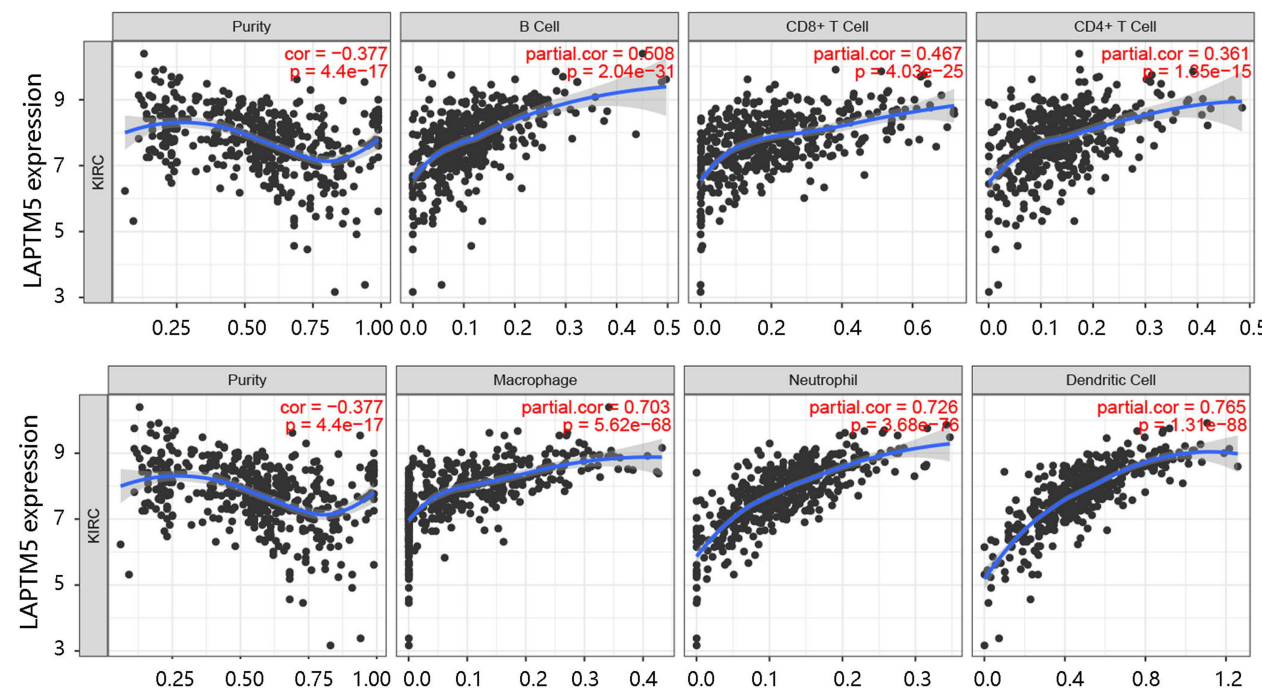

Figure 8 Association of LAPTM5 expression and immune infiltrates.

status of LAPTM5 also affected the prognosis of the patients, and its mRNA expression had a close relation with immune cell infiltration. Therefore, LAPTM5 may be a reliable and potent biomarker for the patient prognosis in KIRC.

\section{Data Sharing Statement}

The datasets used and/or analyzed during the current study are available from the corresponding author on reasonable request.

\section{Ethics Approval}

The Ethics Committee of the Yuyao people's Hospital of Zhejiang Province deemed that this research is based on opensource data, so the need for ethics approval was waived.

\section{Disclosure}

The authors declare that they have no competing interests in this work.

\section{References}

1. Siegel RL, Miller KD, Jemal A. Cancer statistics, 2019. CA Cancer J Clin. 2019;69(1):7-34. doi:10.3322/caac.21551

2. Joosten SC, Smits KM, Aarts MJ, et al. Epigenetics in renal cell cancer: mechanisms and clinical applications. Nat Rev Urol. 2018;15(7):430-451. doi:10.1038/s41585-018-0023-z

3. Hsieh JJ, Purdue MP, Signoretti S, et al. Renal cell carcinoma. Nat Rev Dis Primers. 2017;3:17009. doi:10.1038/nrdp.2017.9

4. Lai Y, Zeng T, Liang X, et al. Cell death-related molecules and biomarkers for renal cell carcinoma targeted therapy. Cancer Cell Int. 2019;19:221. doi:10.1186/s12935-019-0939-2

5. Iacovelli R, Verzoni E, Braud F, et al. First line treatment of metastatic renal cell carcinoma: two standards with different toxicity profile. Cancer Biol Ther. 2014;15(1):19-21. doi:10.4161/cbt.27150

6. Dagogo-Jack I, Shaw AT. Tumour heterogeneity and resistance to cancer therapies. Nat Rev Clin Oncol. 2018;15(2):81-94. doi:10.1038/ nrclinonc.2017.166

7. Santoni M, Pantano F, Amantini C, et al. Emerging strategies to overcome the resistance to current mTOR inhibitors in renal cell carcinoma. Biochim Biophys Acta. 2014;1845(2):221-231. doi:10.1016/j.bbcan.2014.01.007

8. Pak Y, Glowacka WK, Bruce MC, et al. Transport of LAPTM5 to lysosomes requires association with the ubiquitin ligase Nedd4, but not LAPTM5 ubiquitination. J Cell Biol. 2006;175(4):631-645. doi:10.1083/jcb.200603001

9. Li X, Su Y, Zhang J, et al. LAPTM5 plays a key role in the diagnosis and prognosis of testicular germ cell tumors. Int J Genomics. 2021;2021:8816456. doi:10.1155/2021/8816456

10. Glowacka WK, Alberts P, Ouchida R, et al. LAPTM5 protein is a positive regulator of proinflammatory signaling pathways in macrophages. $J$ Biol Chem. 2012;287(33):27691-27702. doi:10.1074/jbc.M112.355917

11. Adra CN, Zhu S, Ko J-L, et al. LAPTM5: a novel lysosomal-associated multispanning membrane protein preferentially expressed in hematopoietic cells. Genomics. 1996;35(2):328-337. doi:10.1006/geno.1996.0364 
12. Wang X, Trotman LC, Koppie T, et al. NEDD4-1 is a proto-oncogenic ubiquitin ligase for PTEN. Cell. 2007;128(1):129-139. doi:10.1016/j. cell.2006.11.039

13. Seimiya M, O-Wang J, Bahar R, et al. Stage-specific expression of Clast6/E3/LAPTM5 during B cell differentiation: elevated expression in human B lymphomas. INT J ONCOL. 2003;22(2):301-304.

14. Kawano Y, Ouchida R, Wang J-Y, et al. A novel mechanism for the autonomous termination of pre-B cell receptor expression via induction of lysosome-associated protein transmembrane 5. Mol Cell Biol. 2012;32(21):4462-4471. doi:10.1128/MCB.00531-12

15. Zhang H, Wei Q, Liu R, et al. Overexpression of LAPTM4B-35: a novel marker of poor prognosis of prostate cancer. PLoS One. 2014;9(3):e91069. doi:10.1371/journal.pone.0091069

16. Xiao M, Jia S, Wang H, et al. Overexpression of LAPTM4B: an independent prognostic marker in breast cancer. J Cancer Res Clin Oncol. 2013;139(4):661-667. doi:10.1007/s00432-012-1368-y

17. Shao GZ, Zhou R-L, Zhang Q-Y, et al. Molecular cloning and characterization of LAPTM4B, a novel gene upregulated in hepatocellular carcinoma. Oncogene. 2003;22(32):5060-5069. doi:10.1038/sj.onc.1206832

18. Sui Y, Lu K, Fu L. Prediction and analysis of novel key genes ITGAX, LAPTM5, SERPINE1 in clear cell renal cell carcinoma through bioinformatics analysis. PeerJ. 2021;9:e11272. doi:10.7717/peerj.11272

19. Xiong Y, Wei Y, Gu Y, et al. DiseaseMeth version 2.0: a major expansion and update of the human disease methylation database. Nucleic Acids Res. 2017;45(D1):D888-D895. doi:10.1093/nar/gkw1123

20. Xu Y, Wu G, Li J, et al. Screening and identification of key biomarkers for bladder cancer: a study based on TCGA and GEO data. Biomed Res Int. 2020;2020:8283401. doi:10.1155/2020/8283401

21. Xu Y, Li X, Han Y, et al. A new prognostic risk model based on PPAR pathway-related genes in kidney renal clear cell carcinoma. PPAR Res. 2020;2020:6937475. doi:10.1155/2020/6937475

22. Nuylan M, Kawano T, Inazawa J, et al. Down-regulation of LAPTM5 in human cancer cells. Oncotarget. 2016;7(19):28320-28328. doi:10.18632/ oncotarget.8614

23. Chen L, Wang G, Luo Y, et al. Downregulation of LAPTM5 suppresses cell proliferation and viability inducing cell cycle arrest at G0/G1 phase of bladder cancer cells. Int J Oncol. 2017;50(1):263-271. doi:10.3892/ijo.2016.3788

24. Cortese R, Hartmann O, Berlin K, et al. Correlative gene expression and DNA methylation profiling in lung development nominate new biomarkers in lung cancer. Int J Biochem Cell Biol. 2008;40(8):1494-1508. doi:10.1016/j.biocel.2007.11.018

25. Inoue J, Misawa A, Tanaka Y, et al. Lysosomal-associated protein multispanning transmembrane 5 gene (LAPTM5) is associated with spontaneous regression of neuroblastomas. PLoS One. 2009;4(9):e7099. doi:10.1371/journal.pone.0007099

26. Hayami Y, Iida S, Nakazawa N, et al. Inactivation of the E3/LAPTm5 gene by chromosomal rearrangement and DNA methylation in human multiple myeloma. Leukemia. 2003;17(8):1650-1657. doi:10.1038/sj.leu.2403026

27. Bagratuni T, Mavrianou N, Gavalas NG, et al. JQ1 inhibits tumour growth in combination with cisplatin and suppresses JAK/STAT signalling pathway in ovarian cancer. Eur J Cancer. 2020;126:125-135. doi:10.1016/j.ejca.2019.11.017

International Journal of General Medicine

Dovepress

\section{Publish your work in this journal}

The International Journal of General Medicine is an international, peer-reviewed open-access journal that focuses on general and internal medicine, pathogenesis, epidemiology, diagnosis, monitoring and treatment protocols. The journal is characterized by the rapid reporting of reviews, original research and clinical studies across all disease areas. The manuscript management system is completely online and includes a very quick and fair peer-review system, which is all easy to use. Visit http://www.dovepress.com/testimonials.php to read real quotes from published authors.

Submit your manuscript here: https://www.dovepress.com/international-journal-of-general-medicine-journal 